\title{
Dermatological Manifestations of COVID-19 in Children
}

\author{
Vivek Athwani ${ }^{1}$ and Sunil Gothwal ${ }^{1, *}$ \\ ${ }^{1}$ Department of Paediatrics, SMS Medical College and J K Lon Hospital Jaipur, Rajasthan, India \\ "Corresponding author: SMS Medical College and J K Lon Hospital Jaipur, Rajasthan, India. Fax: +91-1412619827, Email: dr.sunilgothwal@gmail.com \\ Received 2020 July 09; Accepted 2020 August 01.
}

\begin{abstract}
Coronavirus infection 2019 (COVID-19) primarily has a respiratory system and multi-systemic involvement. Respiratory and gastrointestinal symptoms are predominantly seen in children. In adults, few COVID-19 cases are reported with cutaneous manifestations. Although children are less severely affected by COVID-19, there is increasing evidence for skin involvement, which is in the form of chilblain (e.g., lesions, vesicular, and maculopapular) and erythema multiforme (e.g., rash). Also, few COVID-19 cases are presented with a clinical picture of atypical Kawasaki disease and toxic shock syndrome, later defined as pediatric multisystem inflammatory syndrome (PMIS). The present study aims to summarize various skin lesions with COVID-19.
\end{abstract}

Keywords: Skin, Children, COVID-19

\section{Context}

Coronavirus disease has impacted the whole world in a short time. WHO has identified SARS-CoV2 as a fundamental cause of this outbreak (1). The burden of COVID-19 children was $2.9 \%$ and $1.7 \%$ in China and the United States, respectively. Most children with SARS-CoV2 have either asymptomatic infection or mild illnesses. Reportedly, 2.5\% of pediatric cases in China had severe illnesses (2).

In a meta-analysis, Chang et al. (3) found that 98\% of children had mild to moderate diseases with the main symptoms of fever, cough, fatigue, and gastrointestinal problems. Asymptomatic children can play a significant role in spreading the COVID-19 in the community as they may shed the virus in respiratory secretions and possibly in feces (4). Skin manifestations have recently been the center of attention because they can facilitate COVID-19 diagnosis.

Recalcati first reported the presence of cutaneous manifestation in COVID-19 (5). Since then, a few case reports (5-9) and case series (10) have identified a range of potential dermatological manifestations of COVID-19 in adults. Similarly, in case reports (11-15), children who complained about skin rashes became COVID-19 positive. On the other hand, in Europe and North America (16-18), a group of children was hospitalized in intensive care units with a clinical picture of atypical Kawasaki disease (KD) and toxic shock syndrome, and few of them tested positive for COVID-19. These cases were labeled as Pediatric multisystem inflammatory syndromes (PMIS), a COVID-19 associated condi- tion, including inflammation, fever, and multiple organ failure (19).

\section{Cutaneous Manifestations in COVID-19}

Cutaneous manifestations in viral diseases usually show a typical but variable pattern in COVID-19. Recent case series from Spain $(\mathrm{n}=375)$ described five significant clinical skin patterns in COVID-19 children, as mentioned below. A few patients showed other minor manifestations such as enanthem or purpuric flexural lesions. The main skin patterns include 1. Chilblains like lesions (Figure 1) - acral erythema with vesicles or pustules, 2. Vesicular eruptions (Varicella like), 3. Maculopapular Eruptions, 4. Urticaria, and 5. Livedo/ Necrosis.

\section{Cutaneous Manifestations in Children During COVID-19}

Pseudochilblain or Pernio-like acral lesions are common among children. Docampo-Simon et al. observed a chilblain-like lesion in the COVID-19 pandemic. The median age of presentation was 14 years (3 month - 85 years) with no sex predilection (6). Classic chilblains are cold-induced, benign, self-limited, and involve erythema/ swelling of the toes and fingers. These bright red/purple color fingers or toes in children are termed COVID toes. 


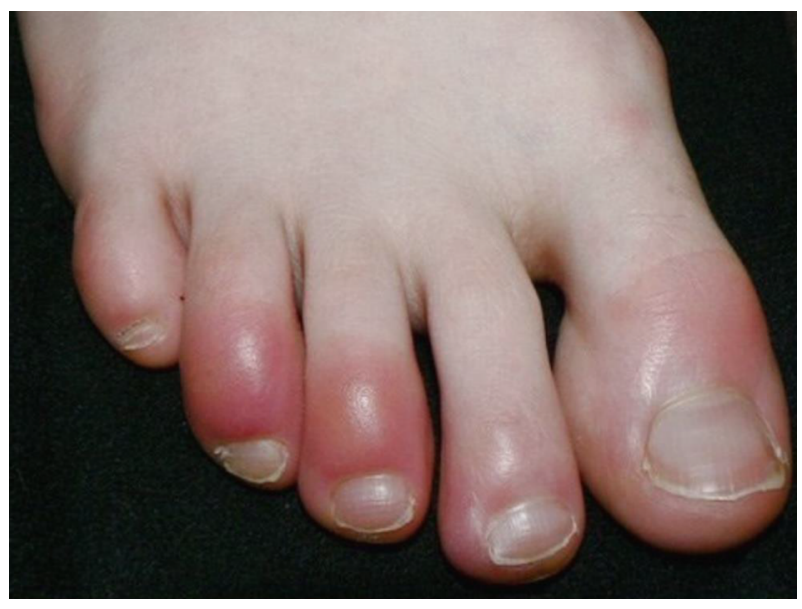

Figure 1. Chilblain like lesion (COVID Toes)

These COVID-19 skin lesions appear several days after systemic symptoms. These children often remain asymptomatic, and the tests for active COVID-19 infection are negative at the time of the first clinical evaluation of cutaneous manifestation (14). Recalcati et al. observed 11 asymptomatic or mildly symptomatic children with $13-18$ years of age with acral-cutaneous pernio-like lesion on their feet and, or hands. Etiology was excluded for other common causes as Ebstein-Barr virus, cytomegalovirus, and parvo B19 virus. Although these children tested negative for SARSCoV-2, Recalcati et al. suspected that the skin manifestations may be related to the COVID-19 pandemic. This hypothesis is strongly supported due to the clustering of unusual skin lesions, the occurrence of familial cases despite restrictions and reporting of similar cases from other parts of affected areas in parallel with pandemic diffusion. The swab negativity could be explained by the disappearance of detectable viral presence after a brief asymptomatic course. According to this hypothesis, the observed skin lesions can represent late manifestations of the COVID-19 infection in young, healthy subjects, possibly due to an immunologic response (7). Colonna et al. reported four similar cases; punch biopsy of the lesion in an 11-year-old girl had dense lymphocytic perivascular and periadnexal infiltrates extending to the subcutis. The signs of vasculitis were evident in small to medium-sized vessels with endothelial cell swelling and red blood cell extravasation. The clinical finding of acro-ischemia and vasculitis may not be an unusual manifestation of COVID-19 infection (14).

SARS-CoV-2 PCR was positive only in few cases with these lesions. The test was neither taken due to the burden of the pandemic nor the policy of the concerned region. Since PCR might be harmful due to presenting late in the disease course, antibody-based tests might be useful. If there were no apparent causes, these children would be tested for SARS-CoV-2 by PCR or by serology if lesions lasted for $>4$ weeks (11). Lesions are less commonly reported from tropical countries due to vascular hyper-responsiveness to cold, resulting in cutaneous inflammation or change in the COVID-19-related thrombotic pattern and endothelial damage. Although there are no treatment guidelines for COVID-19-related, pernio-like lesions of the feet or hands, high-potency topical corticosteroids may be helpful if the lesions are causing discomfort. Severe cases of pernio-like lesions were reported, which had a differential diagnosis with insect or snake bite. These lesions could be found in children with few symptoms $(7,11,14)$ and the adult patients. They might have elevated D-dimer and deranged coagulation profile, which may be the expression of microthrombosis due to endothelial damage and vascular abnormalities (9).

In two of the three case reports by Guarnari et al. (20), two 14-year-old boys initially presented with perenio-like rashes. Since their family tested SARS-CoV-2 positive, they were also SARS-CoV-2 positive. In the third case, there was an 18-year-old boy who developed similar lesions on the acral part of feet with fever $\left(38.5^{\circ} \mathrm{C}\right)$, and skin manifestations emerged after ten days. He had a history of close contact with his COVID-19 positive grandfather. The differential diagnosis of these pediatric COVID-19 skin lesions included cold-induced skin injury, adverse drug reaction, Ebstein-Barr virus, cytomegalo virus, and Parvo B19 virus infection. Genovese et al. reported 22 cases (21 adults \& 1 child), including one Italian 8-year-old girl with varicella similar to exanthema in COVID-19 cases. Although Papulovesicular exanthema appeared after three days of cough and lasted for seven days, these rashes were not associated with typical symptoms of varicella, such as pruritus, pain, or burning sensation (15).

Morey-Olivé et al. reported a case of a two-month-old girl presented with fever and pruritic urticarial rash of 4 days duration. These rashes were present all over except for palms and soles. She had a history of contact with confirmed COVID-19, so she was tested positive for SARS-CoV2 PCR. These lesions were completely resolved in five days (21). In adults, urticarial rashes were presented with the onset of classic symptoms involved mainly trunk and was associated with severe form of COVID-19 (10). Treatment is usually symptomatic with anti-histaminics. The differential diagnosis is a drug-induced rash or acute idiopathic urticaria.

Morey-Olivé et al. also reported a case of a six-year-old boy initially hospitalized for the evaluation of cholestatic liver disease. After two weeks, his clinical symptoms worsened, and he tested positive for SARS-CoV-2. After two days, 
he developed a maculopapular, erythematous, confluent, nonpruritic rash with onset in the chest and neck spreading to the whole body. These skin manifestations were resolved in five days with an improvement in the other symptoms (cholestasis) without specific treatment. The boy did not show any classical symptoms of coronavirus infection (21). In Spain, Galvan Casas C et al. observed maculopapular rashes in $47 \%$ cases of COVID19 patients with skin manifestations. These rashes were usually accompanied by other symptoms and had more severe COVID-19, more than 50\% of which had pruritus (10).

Duramaz et al. (12) from Turkey reported that 15\% of their young patients developed cutaneous manifestations. Their youngest patient was an eight-month-girl with erythematous skin rash similar to roseola associated with fever, which was disappeared in two days. Another patient was an eleven-year-girl who had maculopapular rashes disappeared in five days. Also, a seventeen-yearold teenager had similar rashes after three days of treatment with hydroxyl-chloroquine (HCQ), which was disappeared after stopping the drug. In the case of this boy, it was difficult to decide whether the rashes were due to disease or drug. According to Olisova et al. (13), a girl who tested COVID-19 positive after two days of fever had erythematous macular rashes and purpuric eruptions on the upper eyelid and temporal region associated with the disappearance of fever. Also, her tongue was slightly swollen with prominent lingual papillae. These eruptions disappeared in three days without treatment. These vesicular, maculopapular, and purpuric rashes might have a direct cytopathic effect of SARS-CoV-2 or an indirect cytopathic effect stimulated by cytokines. Differential diagnoses include other viral illnesses, such as measles, Epstein Barr virus, and drug-induced exanthema (22).

A fifteen-day-old term neonate was admitted with fever and mottling. He had no cough, runny nose, or gastrointestinal symptoms. On examination, he was vigilant with tachycardia, tachypnoea (respiratory rate of 66), and mild subcostal retraction, $\mathrm{O}_{2}$ saturation was $93 \%$ (without oxygen). Blood glucose, arterial blood gases, and chest X-ray were normal. Septicemia was ruled out as sepsis screen and blood, urine, and stool culture were negative. RT-PCR for COVID-19 was positive. In this case, the mottling was attributed to COVID-19 as septicemia was ruled out. Per local policy test was conducted for parents, but the child was tested for coronavirus and influenza. The child tested positive for COVID-19 and negative for influenza (23). Some infants born to COVID-19 positive mothers had transient rashes. These were maculopapular rashes to miliria-like eruption, which were disappeared in a few days without treatment (24). Erythema Multiforme (EM)-like lesion was less frequently seen in adults and children. In a case se- ries by Recalcati et al. (7), two children developed erythematopapular lesions on forearms after initial chilblain-like lesions were resolved spontaneously.

Torrelo et al. reported erythema multiforme (EM)-like lesions in pediatric chilblains related cases in his study. They found no specific cause for these lesions, one of which tested positive for SARS-CoV-2 PCR. There was perivascular, perieccrine infiltrate, and no necrosis of keratinocytes in skin biopsies of two patients. These features were not typical in EM. Immunohistochemistry for the above biopsies showed granular positivity in endothelial cells and epithelial cells of eccrine glands. These patients fully improved in one to three weeks without complications (25).

We observed typical dengue fever-like petechial/erythematous purpuric lesions in COVID-19 patients who had mild symptoms due to thrombogenic vasculopathy, the deposition of complement components, or COVID-19 spike glycoproteins (8). Differential diagnoses include rashes due to other viral diseases, such as dengue, scrub typhus, and drug-induced rash. Similarly, livido/necrosis was not reported in children. These manifestations may be explained by disseminated intravascular coagulation and deoxygenated venous blood due to hypoxia (10).

Galvan Casas et al. attempted to categorize skin lesions with the severity of illness. Disease severity had a pattern of less severe disease in pseudo-chilblain to more severe in livedoid presentations in the form of pneumonia, hospitalization, and intensive care requirements (10).

In PMIS, children are reported to have atypical or classical KD or toxic shock syndrome, such as polymorphic rashes (16-18, 26, 27). Atypical or Classical Kawasaki disease was previously reported to be associated with inciting infectious disease due to the sudden rise of the COVID-19 pandemic (28). The WHO and CDC had proposed the case definition for PMIS (19).

Despite the unknown mechanism of skin manifestations, we proposed the role of the immune response, complement system, and microvascular injury. According to the pathomechanisms proposed by Suchonwanit et al., the skin manifestations are classified into two significant types: (a) viral exanthems (b) vasculopathy: vasculitis and thrombotic vasculopathy (29). Eosinophilia (drugrelated), with systemic symptoms, is a crucial differential diagnosis. Treatment options are antibiotics and antiviral drugs.

Clinical photographs and histological confirmation of skin lesions in COVID pediatric cases were not possible due to limited resources like personal protective equipment and manpower. Given that the evidencebased science of COVID-19 and its skin manifestation are at the early stage, it is crucial to report cutaneous 
manifestations in the present condition because it may help us better understand and diagnose the disease. http://www.aad.org/covidregistry is an available online registry for recording and understanding the COVID-19 skin manifestations (30).

\section{Conclusions}

Although cutaneous manifestations in COVID-19 are non-specific, pseudo-chilblain and vesicular lesion may currently be a useful indicator of the COVID-19 pandemic. Identifying the skin markers for COVID-19 in mild or asymptomatic patients may be helpful as an epidemiological marker rather than for diagnosis.

Any unusual rash or typical rash with non-classical findings of other illnesses such as varicella and dengue may be associated with COVID-19. During this time, testing for SARS-CoV-2 is conducted in case of any skin alterations, which can be useful in early diagnosis of COVID-19 to break the chain of transmission. Significantly, the clinicians should be conscious of COVID-19-related skin manifestation in order to minimize misdiagnosis. They should also differentiate if lesions are caused by COVID-19 or secondary to drugs.

\section{Footnotes}

Authors' Contribution: SG and VA participated in the study concept and design, analysis and interpretation of literature, drafting of the manuscript, and critical revision of the manuscript. Also, SG supervised the study overall.

\section{Conflict of Interests: None.}

Funding/Support: None.

\section{References}

1. WHO. COVID-19 dashboard. Geneva: World Health Organization; 2020 [cited 12 Jun 2020]. Available from: https://who.sprinklr.com/.

2. Dong Y, Mo X, Hu Y, Qi X, Jiang F, Jiang Z, et al. Epidemiology of COVID-19 among children in China. Pediatrics. 2020;145(6). doi 10.1542/peds.2020-0702. [PubMed: 32179660].

3. Chang TH, Wu JL, Chang LY. Clinical characteristics and diagnostic challenges of pediatric COVID-19: A systematic review and meta-analysis. J Formos Med Assoc. 2020;119(5):982-9. doi: 10.1016/j.jfma.2020.04.007. [PubMed: 32307322]. [PubMed Central: PMC7161491].

4. Kelvin AA, Halperin S. COVID-19 in children: the link in the transmission chain. Lancet Infect Dis. 2020;20(6):633-4. doi: 10.1016/S1473-3099(20)30236-X. [PubMed: 32220651]. [PubMed Central: PMC7156154].

5. Recalcati S. Cutaneous manifestations in COVID-19: a first perspective. J Eur Acad Dermatol Venereol. 2020;34(5):e212-3. doi: 10.1111/jdv.16387. [PubMed: 32215952].
6. Docampo-Simon A, Sanchez-Pujol MJ, Juan-Carpena G, PalazonCabanes JC, Vergara-De Caso E, Berbegal L, et al. Are chilblain-like acral skin lesions really indicative of COVID-19? A prospective study and literature review. J Eur Acad Dermatol Venereol. 2020. doi: 10.1111/jdv.16665. [PubMed: 32452545]. [PubMed Central: PMC7283627].

7. Recalcati S, Barbagallo T, Frasin LA, Prestinari F, Cogliardi A, Provero MC, et al. Acral cutaneous lesions in the time of COVID-19. J Eur Acad Dermatol Venereol. 2020;34(8):e346-7. doi: 10.1111/jdv.16533. [PubMed: 32330324]. [PubMed Central: PMC7267354].

8. Joob B, Wiwanitkit V. COVID-19 can present with a rash and be mistaken for dengue. J Am Acad Dermatol. 2020;82(5). e177. doi: 10.1016/j.jaad.2020.03.036. [PubMed: 32213305]. [PubMed Central: PMC7156802].

9. Suarez-Valle A, Fernandez-Nieto D, Diaz-Guimaraens B, DominguezSantas M, Carretero I, Perez- Garcia B. Acro-ischaemia in hospitalized COVID-19 patients. J Eur Acad Dermatol Venereol. 2020;34(9). doi: 10.1111/jdv.16592.

10. Galvan Casas C, Catala A, Carretero Hernandez G, Rodriguez-Jimenez P, Fernandez-Nieto D, Rodriguez-Villa Lario A, et al. Classification of the cutaneous manifestations of COVID-19: a rapid prospective nationwide consensus study in Spain with 375 cases. Br J Dermatol. 2020;183(1):71-7. doi: 10.1111/bjd.19163. [PubMed: 32348545]. [PubMed Central: PMC7267236].

11. Mazzotta F, Troccoli T. Acute acro-ischemia in the child at the time of COVID-19. Eur J Pediat Dermatol. 2020;30(2):71-4. doi: 10.26326/22819649.30.2.2102.

12. Bursal Duramaz B, Yozgat CY, Yozgat Y, Turel O. Appearance of skin rash in pediatric patients with COVID-19: Three case presentations. Dermatol Ther. 2020;33(4). e13594. doi: 10.1111/dth.13594. [PubMed: 32412681]. [PubMed Central: PMC7261998].

13. Olisova OY, Anpilogova EM, Shnakhova LM. Cutaneous manifestations in COVID-19: A skin rash in a child. Dermatol Ther. 2020. e13712. doi: 10.1111/dth.13712. [PubMed: 32472593]. [PubMed Central: PMC7300520].

14. Colonna C, Monzani NA, Rocchi A, Gianotti R, Boggio F, Gelmetti C. Chilblain-like lesions in children following suspected COVID-19 infection. Pediatr Dermatol. 2020;37(3):437-40. doi: 10.1111/pde.14210. [PubMed: 32374033]. [PubMed Central: PMC7267284].

15. Genovese G, Colonna C, Marzano AV. Varicella-like exanthem associated with COVID-19 in an 8-year-old girl: A diagnostic clue? Pediatr Dermatol. 2020;37(3):435-6. doi: 10.1111/pde.14201. [PubMed: 32315079]. [PubMed Central: PMC7264614].

16. Toubiana J, Poirault C, Corsia A, Bajolle F, Fourgeaud J, Angoulvant F, et al. Kawasaki-like multisystem inflammatory syndrome in children during the covid-19 pandemic in Paris, France: prospective observational study. BMJ.2020;369:m2094.doi:10.1136/bmj.m2094. [PubMed: 32493739].

17. Verdoni L, Mazza A, Gervasoni A, Martelli L, Ruggeri M, Ciuffreda M, et al. An outbreak of severe Kawasaki-like disease at the Italian epicentre of the SARS-CoV-2 epidemic: an observational cohort study. Lancet 2020;395(10239):1771-8. doi: 10.1016/S0140-6736(20)31103-X. [PubMed: 32410760]. [PubMed Central: PMC7220177].

18. Riphagen S, Gomez X, Gonzalez-Martinez C, Wilkinson N, Theocharis P. Hyperinflammatory shock in children during COVID-19 pandemic Lancet. 2020;395(10237):1607-8. doi: 10.1016/S0140-6736(20)31094-1. [PubMed: 32386565]. [PubMed Central: PMC7204765].

19. CDC. Multisystem inflammatory syndrome in children (MIS-C) associated with coronavirus disease 2019(COVID-19). Atlanta: center of disease control; 2020. Available from: https://www.cdc.gov/coronavirus/2019ncov/daily-life-coping/children/mis-c.html.

20. Guarneri C, Rullo EV, Pavone P, Berretta M, Ceccarelli M, Natale A, et al. Silent COVID-19: what your skin can reveal. Lancet Infect Dis. 2020 doi: 10.1016/S1473-3099(20)30402-3. [PubMed: 32437697]. [PubMed Central: PMC7234773]. 
21. Morey-Olive M, Espiau M, Mercadal-Hally M, Lera-Carballo E, GarciaPatos V. Cutaneous manifestations in the current pandemic of coronavirus infection disease (COVID 2019). An Pediatr (Engl Ed) 2020;92(6):374-5. doi: 10.1016/j.anpede.2020.04.002. [PubMed: 32341944]. [PubMed Central: PMC7183933].

22. Sachdeva M, Gianotti R, Shah M, Bradanini L, Tosi D, Veraldi S, et al. Cutaneous manifestations of COVID-19: Report of three cases and a review of literature. J Dermatol Sci. 2020;98(2):75-81. doi 10.1016/j.jdermsci.2020.04.011. [PubMed: 32381430]. [PubMed Central: PMC7189855].

23. Kamali Aghdam M, Jafari N, Eftekhari K. Novel coronavirus in a 15-day-old neonate with clinical signs of sepsis, a case report. In fect Dis (Lond). 2020;52(6):427-9. doi: 10.1080/23744235.2020.1747634. [PubMed: 32233816]. [PubMed Central: PMC7157949].

24. Chen Y, Peng H, Wang L, Zhao Y, Zeng L, Gao H, et al. Infants born to mothers with a new coronavirus (COVID-19). Front Pediatr. 2020;8:104 doi: 10.3389/fped.2020.00104. [PubMed: 32266184]. [PubMed Central: PMC7098456]

25. Torrelo A, Andina D, Santonja C, Noguera-Morel L, Bascuas-Arribas M, Gaitero-Tristan J, et al. Erythema multiforme-like lesions in children and COVID-19. Pediatr Dermatol. 2020;37(3):442-6. doi: 10.1111/pde.14246. [PubMed: 32445583]. [PubMed Central
PMC7283638].

26. Jones VG, Mills M, Suarez D, Hogan CA, Yeh D, Segal JB, et al. COVID19 and kawasaki disease: Novel virus and novel case. Hosp Pediatr. 2020;10(6):537-40. doi:10.1542/hpeds.2020-0123. [PubMed: 32265235].

27. Yozgat CY, Uzuner S, Bursal Duramaz B, Yozgat Y, Erenberk U, Iscan A, et al. Dermatological manifestation of pediatrics multisystem inflammatory syndrome associated with COVID-19 in a 3-year-old girl. Dermatol Ther. 2020;33(4). e13770. doi: 10.1111/dth.13770. [PubMed: 32500647]. [PubMed Central: PMC7300549].

28. Principi N, Rigante D, Esposito S. The role of infection in Kawasaki syndrome. J Infect. 2013;67(1):1-10. doi: 10.1016/j.jinf.2013.04.004. [PubMed: 23603251]. [PubMed Central: PMC7132405].

29. Suchonwanit P, Leerunyakul K, Kositkuljorn C. Cutaneous manifestations in COVID-19: Lessons learned from current evidence. $J$ Am Acad Dermatol. 2020;83(1):e57-60. doi: 10.1016/j.jaad.2020.04.094. [PubMed: 32339706]. [PubMed Central: PMC7194618].

30. Freeman EE, McMahon DE, Fitzgerald ME, Fox LP, Rosenbach M, Takeshita J, et al. The American Academy of Dermatology COVID19 registry: Crowdsourcing dermatology in the age of COVID-19. J Am Acad Dermatol. 2020;83(2):509-10. doi: 10.1016/j.jaad.2020.04.045. [PubMed: 32305438]. [PubMed Central: PMC7162762]. 